\title{
WORKFORCE Principles and practice of deploying a flexible physician workforce for COVID-19 care wards from a Dutch hospital
}

\author{
Authors: Marcel Spanier, ${ }^{A}$ Katja Kerkvliet ${ }^{B}$ and Anke Veeman ${ }^{C}$
}

\begin{abstract}
During the COVID-19 pandemic, the attainment of the required hospital workforce is extremely challenging; with a requirement to create surge capacity for COVID-19 care and to ensure and sustain regular hospital care. The ongoing COVID-19 pandemic demands the deployment of a flexible physician workforce for the cohort wards in hospitals. This can be successfully be organised and managed if some key principles are followed. Physicians have the ability to practice their general, not specialised, medical skills. COVID-19 specific medical education, re- and upskilling can be necessary. This approach for the future physician workforce may be applicable in several national healthcare systems. Solidarity, flexibility and transparency within the medical staff are the cornerstones for a successful deployment of a physician flex pool in a hospital dealing with a pandemic.
\end{abstract}

KEYWORDS: COVID-19, workforce, capacity

DOI: $10.7861 /$ fhj.2020-0254

\section{Introduction}

As a third wave of the COVID-19 pandemic progresses, the attainment of the necessary hospital workforce remains extremely challenging; with a requirement to create surge capacity for COVID-19 care and to ensure elective hospital care workforce for as long as possible. ${ }^{1-3}$ Unfortunately, in many hospitals, the non-urgent medical care has had to be downscaled and the acute care for COVID-19 patients has to be increased. Hospital care for COVID-19 patients is organised on cohort wards and intensive care cohort units. The healthcare workers on these cohort wards work under great pressure. ${ }^{4}$ There is a need to replace staff, when they are being quarantined or are away from work because of work stress. Ultimately, there is a need for a flexible and appropriately skilled physician workforce for the COVID-19 care wards

Authors: ${ }^{\text {A }}$ consultant gastroenterologist, Rijnstate Hospital, Arnhem, the Netherlands; ${ }^{B}$ hospital pharmacy consultant, Rijnstate Hospital, Arnhem, the Netherlands; ' medical staff director, Rijnstate Hospital, Arnhem, the Netherlands
In our Dutch large teaching hospital, we instituted a physician flex pool during the first COVID-19 wave to fulfil the surge workforce capacity for the COVID-19 care wards. The coordination of the physician workforce was performed by the medical staff. The organisation and functioning of the flex pool were evaluated and reinstalled during the second COVID-19 wave. We describe the key principles and practice of deploying such a flexible physician workforce for the COVID-19 care wards developed from learning from the first wave.

\section{Principles of a flexible physician workforce Core staffing requirement of COVID-19 cohort wards}

The required physician capacity per scaled-up cohort ward is determined by the representatives of the resident and staff members of the infectious diseases and respiratory medicine together with geriatricians. In our hospital, a cohort ward consists of 12-15 beds for proven or highly suspected COVID-19 patients. Per cohort ward, two ward physicians and one supervising physician are needed for a day shift. For every two cohort wards, one extra ward physician is needed for the evening shift. The night shift is performed by residents from internal and pulmonary medicine. If there are three or more COVID-19 cohort wards, an extra resident per night shift is required. The required nurse capacity and deployment is separately coordinated by managers appointed by the hospital board.

Our teaching hospital has 766 inpatient beds. During the first wave, a maximum of five COVID-19 wards were installed for 60 COVID-19 patients. Normally, the intensive care unit has 14 beds and was upscaled to 28 beds: 24 for COVID-19 patients and four for non-COVID care. Our medical staffing, with 26 different specialisms, consists of approximately 315 medical staff members and 270 residents. Almost all specialties participated in the flex pool.

\section{Training in management of COVID-19}

A distinction is made between the ward physician position and supervisor role. The ward physician role is deployed by junior and senior residents from both internal and surgical specialisms. There was not a specific time period for deployment of the residents with general medical experience. Supervising physicians are currently staff members of the non-surgical specialisms. To 
ensure the correct clinical skills for the deployed ward physicians, all residents with an ongoing or prior training in internal medicine are suitable for this role. If needed, reskilling in acute medicine is organised. Residents and attending surgical staff can be made competent with additional education. We observed that during the second COVID-19 wave more expertise of internal medicine is needed as the admitted COVID-19 patients have more general internal and oncological comorbidities. Every new ward physician and supervisor follows a 3-day course on the COVID-19 ward and then works under direct supervision of an experienced COVID-19 ward physician. The staff of infectious diseases and respiratory medicine are responsible for the COVID-19-specific medical treatment protocols and supervise all COVID-19 wards. E-learning in COVID-19 care is also made available. General practitioners in training doing their clinical internship are also suitable. Physicians (such as radiologists and pathologists) are not primarily used for deployment on the cohort wards. They are deployed as coordinators for the outplacement of COVID-19 patients. This coordinator is in contact with the regional and national coordination centre for patient distribution and keeps track of the capacity of all Dutch hospitals and intensive care units. Cardiologists and anaesthetists and their residents are deployed on the medium care and intensive care.

\section{Recruitment and deployment across specialties}

Two members of the medical board were appointed as initiators and contact people for the physician flex pool. They initiated meetings and discussions to determine the preconditions as described. They are the key players for organisational questions and concerns of medical staff. The medical board made a general appeal to the various medical department chairmen to make physicians available for the flex pool. It is important to indicate that the physicians must be available without another workforce schedule and are available for a limited period of 2-3 weeks. This is to promote enrolment into the flex pool and makes the education beneficial. The two medical board members organise a weekly meeting together with the planners and representatives of the responsible departments of internal medicine, respiratory diseases and geriatrics. This was to evaluate the coordination and the deployment process; and determine what rostering problems are encountered and how the new deployed ward physicians function. A structured evaluation of the deployment process with, for example, questionnaires or interviews is not performed.

During the first COVID-19 wave, several physicians from outside of the hospital volunteered to help with COVID-19 care. Information about these physicians was collected and registered. These physicians are approached by the medical board as demand increased. This external pool of physicians consists of general practitioners (in training), tropical doctors and retired physicians. Currently, this external pool of physicians is not deployed.

\section{Coordination of workforce deployment}

To plan and coordinate the work, it is important that planners are appointed who understand the work floor. In our hospital, they are residents of internal medicine, respiratory diseases and geriatrics. They plan an online schedule programme. All registrations of available physicians are received centrally at a newly created email address (for example, scheduleCOVID@...). It is important that the planners are not responsible for any problems during scheduling (for example, if physicians made available cannot be deployed). When necessary, the two members of the medical board are brought in.

\section{Decision making of capacity requirements}

The crisis policy team, chaired by the executive board of the hospital, decides what the capacity of COVID-19 cohort beds is required. The needed capacity is adjusted nationally and regionally in agreement with other hospitals. The executive board has appointed two capacity managers who provide advice on the feasibility of the requested COVID-19 bed capacity, which depends on the availability of nurses and medical staff. It also depends on the required capacity for non-COVID-19 care. The capacity managers are in close contact with the two members of the medical board. Based on the required COVID-19 cohort beds, the planners are asked to adjust the work schedules.

\section{Linking with emergency department and COVID-19 intensive care}

The two members of the medical board maintain communication with the emergency and intensive care department about the required workforce capacity of these departments. In our hospital, the intensive care unit organises and schedules their own workforce capacity. However, oversight of their workforce is also done by the two members of the medical board. The cardiology and anaesthetics departments are approached to deliver physicians for the intensive care unit. For the emergency department, surgical residents and general practitioner trainees are called upon. It is important to have a general overview of needed workforce capacity for both the cohort ward and intensive care units. Internal competition for staff must be prevented.

\section{Learning}

We experienced during the first wave that both residents and medical staff still have general, not specialised, medical skills and can be re- and upskilled for the required COVID-19 care. Summarised and structured COVID-19 education is of great importance. The residents and staff can play so-called 'total soccer', in which any outfield player can take over the role of any other player in a team. Our conclusion that 'total soccer' really can be played may be of importance for the future role of the physician in the NHS and other national healthcare systems. In a recent report from Health Education England, it is also stated that the COVID-19 pandemic illustrates how crucial generalist skills are in enabling doctors to be deployed in different specialties to meet patient needs. ${ }^{5}$ Furthermore, Fernandes and colleagues discuss in their report that the role of the future physician in the NHS should be changed. ${ }^{6}$ The needed future workforce should be more blended, multi-professional and flexible, for example. More integrated teams of physicians and medical associated professionals will be required. Therefore, the ability of physicians to practice across the breadth of their knowledge will be crucial for the flexibility of the workforce. The COVID-19 pandemic has thrust these needed changes upon the future physician workforce.

We also learned from the first wave that solidarity within medical staff is the cornerstone for a successful functioning of a physician flex pool. The responsible medical board members must be proactive, rather than reactive, in conversation with the 
medical staff from the different departments. Questions and/or concerns arise about solidarity within medical staff, transparency about commitment by departments and problems of availability if regular (outpatient) care has not yet been sufficiently scaled down. It is also important to share the successes with all those involved, stating explicitly what is going well, especially the flexibility of the medical staff.

Interestingly, the second COVID-19 wave led to a different demand of workforce capacity compared with the first wave. There was a greater continuing stream of COVID-19 patients who needed to be admitted in the hospital. Also, hospitals try much longer to continue the regular non-urgent care for as long as possible. In the near future or when the pandemic is over, it will be very challenging to deliver the (patient) delayed or hospital postponed regular non-urgent care. Remarkably during the first wave, there were less acute non-COVID admissions, such as trauma, and heart and cerebrovascular strokes. Furthermore, the needed capacity on the cohort wards is greater than on the intensive care cohort units. This is probably the result of better treatment outcomes which results in COVID-19 patients staying for longer on the cohort wards and need less care on the intensive care units. The needed medical care on the cohort ward warrants more expertise in internal medicine. The admitted COVID-19 patients have more internal and oncological comorbidities, and may develop diabetes as the result of the more frequent use of dexamethasone.

Finally, more healthcare workers are quarantined and/or getting ill. Generally last year, the COVID-19 test capacity has increased and more healthcare workers can be tested for COVID-19. There is significant COVID-19-related sickness and quarantine need among the physicians. During the first and second wave, approximately $9 \%$ of all medical staff members and $14 \%$ of all residents had a positive COVID-19 PCR test. They had to be quarantined for at least 7 days. Furthermore, during the first COVID-19 wave, a psychological support team is instituted for monitoring the wellbeing of both the nurses and physicians. They contact them on demand and proactively visit the COVID-19 wards and intensive care unit on a regular basis. During the second wave, this support team was reinstalled.

\section{Conclusion}

The ongoing COVID-19 pandemic demands the deployment of a flexible physician workforce for the cohort wards in hospitals. This can successfully be organised and managed if some key principles are followed. We learned that both residents and medical staff have the ability to practice their general, not specialised, medical skills. COVID-19 specific medical education, re- and upskilling can be necessary. This flexible approach may be required for the future physician workforce in several national healthcare systems. Finally, solidarity, flexibility and transparency within the medical staff are the cornerstones for a successful functioning of a physician flex pool in a hospital dealing with an emergency situation, such as the present pandemic.

\section{References}

1 Fraher EP, Pittman P, Frogner BK et al. Ensuring and sustaining a pandemic workforce. N Engl J Med 2020;382:2181-3.

2 Dow AW, DiPiro JT, Giddens J, Buckley P, Santen SA. Emerging from the COVID crisis with a stronger health care workforce. Acad Med 2020;10:1097.

3 Cram P, Anderson ML, Shaughnessy EE. All hands on deck: Learning to "un-specialize" in the COVID-19 Pandemic. J Hosp Med 2020; 15:314-5

4 Vindrola-Padros C, Andrews L, Dowrick A et al. Perceptions and experiences of healthcare workers during the COVID-19 pandemic in the UK. BMJ Open 2020;10:e040503.

5 Health Education England. Future doctor: a co-created vision for the future clinical team. HEE. www.hee.nhs.uk/our-work/future-doctor

6 Fernandes L, FitzPatrick ME, Roycroft M. The role of the future physician: building on shifting sands. Clin Med 2020;20:285-9.

Address for correspondence: Dr Marcel Spanier, Department of Gastroenterology and Hepatology, Rijnstate Hospital, Wagnerlaan 55, 6815 AD Arnhem, the Netherlands. Email: mspanier@rijnstate.nl 